\title{
TU3B-(2)-1
}

\section{MID-IR QUANTUM CASCADE LASERS AND AMPLIFIERS: RECENT DEVELOPMENTS AND APPLICATIONS}

\author{
Mariano Troccoli, Federico Capasso \\ Harvard University \\ 310 Cruft Laboratory, 19 Oxford St, Cambridge, MA 01238, USA \\ ph.: +1-617-495-1934 ; fax: +1-617-495-2875; troccoli@deas.harvard.edu
}

Raffaele Colombelli, Claire Gmachl, Donald Tennant, Deborah L. Sivco, Alfred Y. Cho

Bell Laboratories, Lucent Technologies, 600 Mountain Ave. Murray Hill, NJ 07974

\author{
Oskar J. Painter, Kartik Srinivasan \\ California Institute of Technology, MS 128-95 Pasadena, CA 91128
}

Abstract - This talk will give an overview of the most recent results on the realization of new quantum cascade laser devices and the perspective of their innovative applications in the mid-infrared range of the spectrum.

\section{SUMMARY}

The paper presented here will deal with the use of photonic band gaps in one and two dimensions to produce electrical injection laser sources in the mid-infrared (mid-IR) using quantum cascade technology.

Quantum cascade lasers are one of the most prominent light sources in the mid-IR range of the spectrum, owing to their unipolar character, wavelength versatility, high optical power, and small dimensions. Since their first demonstration in 1994, a great quantity of new phenomena, devices and applications has been shown. The most noteworthy among the recent ones are probably the first realization of a device working in continuous wave at room temperature [1], the attainment of lasing at $\mathrm{THz}$ frequencies [2], and the realization of a supercontinuum source in the 5-8 $\mu \mathrm{m}$ range [3]. These devices open up new possibilities in spectroscopy, free-space optical wireless communications, and sensing.

For most of the applications where these sources are involved, one of the common requirements is to have them working on a single-mode, possibly with a high power and a good beam quality. Recently [4] a quantum cascade MOPA amplifier was demonstrated and used to obtain a high power single mode emission from a DFB-QC laser. The use of such a configuration allowed to obtain an output $0.5 \mathrm{~W}$ of single-mode peak power at low temperatures and $0.25 \mathrm{~W}$ at room temperature. The single mode operation was demonstrated with more than $30 \mathrm{~dB}$ of side mode suppression ratio and the combination of the DFB laser with the tapered amplifier lead to a reduction in the beam divergence down to $12 \mathrm{deg}$. of FWHM in the lateral direction.

Aiming for higher degrees of miniaturization and integration, the use of a two dimensional photonic band-gap device allowed us to achieve surface emitting, microscopic devices with milliwatts of output power and a tunability of the laser wavelength depending on the dimensional parameters of the emitters in the laser arrays. We observed lasing action from hexagonal-symmetry hole pattemed photonic crystal devices based on an intersubband injection active region. The pattem of holes was etched into the semiconductor through the active region down into the substrate, in order to avoid light scattering from the bottom surface of the etched holes. On the other hand, the etching was kept shallow enough in order to prevent over-heating of the device with current injection. The trade-off between these two requirements was met using a surface-plasmon waveguide, where the active region lies directly underneath the top contact and the guided mode has its maximum at the metal-semiconductor interface. In the devices where the photonic band structure was well matched with the gain spectrum, narrow peaks in the electroluminescence spectra were observed and could be tuned by varying the hole diameter and the inter-hole spacing. In a significant fraction of the devices showing this enhanced vertical emission, lasing action was observed. The laser wavelength could also be tuned with the device dimensions. Typically, among the multiple peaks observed 
TU3B-(2)-1

in the sub-threshold spectra, the highest-energy one was the one showing line narrowing and eventually laser action at a threshold of about $4 \mathrm{kA} / \mathrm{cm}^{2}$. Far-field and polarization measurements allowed to unambiguously identify the lasing mode of the PBG structure and the agreement with theoretical predictions was very good.

\section{REFERENCES}

[1] M. Beck, D. Hofstetter, T. Aellen, J. Faist, U. Oesterle, M. Ilegems, E. Gini, and H. Melchior, 'Continuous wave operation of a mid-infrared semicondu ctor laser at room temperature', Science, $295 \mathrm{pp}$. $301-305$ (2002).

[2] R. Koehler, A. Tredicucci, F. Beltram, H. E. Beere, E. H. Linfield, A. G. Davies, D. A. Ritchie, R. G. lotti, and F. Rossi, 'Terahertz semiconductor heterostructure laser', Nature, 417 pp. 156-158 (2002).

[3] C. Gmachl, D. L. Sivco, R. Colombelli, F. Capasso, and A. Y. Cho, 'Ultra-broadband semiconductor laser', Nature, 415 pp. 883-885 (2002).

[4] M. Troccoli, C. Gmachl, F. Capasso, D. L. Sivco and A. Y. Cho, 'Mid-infrared ( $\lambda \approx 7.4 u m)$ quantum cascade laser amplifier for high power, single-mode emission and improved beam quality', Appl. Phys. Lett., 80 pp.4103-4105 (2002). 